\title{
Development of an Ensemble Intelligent Model for Assessing the Strength of Cemented Paste Backfill
}

\author{
Yuantian Sun, ${ }^{1}$ Guichen Li $\mathbb{D}^{1},{ }^{1}$ Junfei Zhang, ${ }^{2}$ Junbo Sun, ${ }^{2}$ and Jiahui Xu ${ }^{1}$ \\ ${ }^{1}$ School of Mines, Key Laboratory of Deep Coal Resource Mining, Ministry of Education of China, \\ China University of Mining and Technology, Xuzhou 221116, China \\ ${ }^{2}$ Department of Civil, Environmental and Mining Engineering, The University of Western Australia, Perth 6009, Australia \\ Correspondence should be addressed to Guichen Li; liguichen@cumt.edu.cn and Qiusong Chen
}

Received 6 February 2020; Accepted 27 March 2020; Published 16 April 2020

Academic Editor: Qiusong Chen

Copyright (c) 2020 Yuantian Sun et al. This is an open access article distributed under the Creative Commons Attribution License, which permits unrestricted use, distribution, and reproduction in any medium, provided the original work is properly cited.

Cemented paste backfill (CPB) is an eco-friendly composite containing mine waste or tailings and has been widely used as construction materials in underground stopes. In the field, the uniaxial compressive strength (UCS) of CPB is critical as it is closely related to the stability of stopes. Predicting the UCS of CPB using traditional mathematical models is far from being satisfactory due to the highly nonlinear relationships between the UCS and a large number of influencing variables. To solve this problem, this study uses a support vector machine (SVM) to predict the UCS of CPB. The hyperparameters of the SVM model are tuned using the beetle antennae search (BAS) algorithm; then, the model is called BSVM. The BSVM is then trained on a dataset collected from the experimental results. To explain the importance of each input variable on the UCS of CPB, the variable importance is obtained using a sensitivity study with the BSVM as the objective function. The results show that the proposed BSVM has high prediction accuracy on the test set with a high correlation coefficient $(0.97)$ and low root-mean-square error $(0.27 \mathrm{MPa})$. The proposed model can guide the design of $\mathrm{CPB}$ during mining.

\section{Introduction}

Cemented paste backfill (CPB) is widely used for mining operations in underground metal mines, in which tailings are normally used as main aggregates and they are mixed with cementitious material and water [1]. CPB is normally filled into the underground stope, and thus, it plays a critical role in supporting the roof and surrounding rock mass after a certain period of dehydration and consolidation [2-5]. Compared with other backfill materials, CPB is an eco-friendly and economic mine composite due to the maximum utilization of mine waste, which attracts much attention these years [6-11].

Filling strength is the most important mechanical parameter that affects filling quality, and unconfined compressive strength (UCS) is the most basic and key parameter to evaluate the filling strength of $\mathrm{CPB}$ [12]. Generally, the UCS of CPB is obtained in the laboratory, similar to the strength evaluation of the concrete. However, when multiple parameters are related to UCS of $\mathrm{CPB}$, experimental measurement is a tedious, time-consuming, and expensive method [13, 14]. Many scholars have put forward many methods to predict the strength of CPB such as empirical formula estimation, numerical simulation, and elastic mechanics analysis $[15,16]$. It should be pointed out that the $\mathrm{CPB}$ is a multiphase composite and the mentioned methods normally cannot obtain accurate prediction results. To accurately predict the UCS of CPB, it is necessary to put forward simple and reliable methods.

Recently, machine learning methods have been widely used for predicting the mechanical properties of construction materials [12,17-23]. The assessment of the strength of CPB by artificial intelligence methods has also been presented. For instance, artificial neural network (ANN) considering influencing variables of $\mathrm{CPB}$ has been used to model the relationship between inputs and outputs [24, 25]. Furthermore, the evolutionary ANN method, namely, ANNbased methods, was proposed for estimating the UCS of $\mathrm{CPB}$, by which the hyperparameters such as the number of 
neurons and the structure of ANN are optimized by some global optimization algorithms, i.e., particle swarm optimization (PSO) and firefly algorithm (FA). Similarly, other normally used machine learning methods such as the random forest algorithm (RF) and RF-based models reported in the literature are also used for predicting the UCS of $\mathrm{CPB}$ [26]. Although the above artificial intelligence methods (ANN, ANN-based, RF, and RF-based) were applied in strength prediction of $\mathrm{CPB}$, they are limited in calculating efficiency and uncertain structures. Besides, there is no intelligent model for the prediction of UCS of CPB considering the overall effect of cement-coarse tailings ratio, solids-water ratio, fine tailings percentage, and curing time.

Therefore, in this paper, the machine learning algorithms, support vector machine (SVM) that has the perfect ability in regression and classification, and an excellent global optimization algorithm, beetle antennae search algorithm (BAS) that is used for selecting hyperparameters of SVM, were combined. Therefore, an evolutionary support vector machine model (BSVM) is proposed. Several contributions to the literature can be concluded as follows:

(1) The support vector machine (SVM) and beetle antennae search (BAS) algorithms were combined to establish the evolutionary support vector machine model;

(2) The strength properties of CPB was analyzed by conducting the experiments considering the key influencing variables, i.e., cement-coarse tailings ratio, solids-water ratio, fine tailings percentage, and curing time

(3) The UCS of CPB was directly estimated by considering the combined effect of four key influencing variables

(4) The sensitive analysis of the mentioned influencing variables of CBP was first analyzed and discussed

\section{Materials and Methods}

2.1. Mechanical Tests. To prepare the CPB specimen, the grain size distribution of tailings and the mineralogical composition are necessary to determine. Thus, a laser diffraction analyzer was utilized for determining the size distribution of coarse tailings and fine tailings. As we can see from Figure 1, there are two different tailings of various sizes. To analyze the influence of fine tailing on the strength of CPB is critical. The Portland cement P.O 32.5R was applied as a binder. The water obtained in this mine was used as the mixing water. According to the field trial tests, coarse tailings-cement ratio (T/C) was set as $4,6,8$, and 10 , and the solids-water ratio $(\mathrm{S} / \mathrm{W})$ was set as $0.68,0.70$, and 0.72 . The fine tailings are as an admixture, and its percentage (FTP) was set as $0 \%, 10 \%, 15 \%$, and $20 \%$. The blinder and aggregates were mixed by using a mixer (UJZ-15) for $5 \mathrm{~min}$. Then, the prepared mixture was poured into the molds $(70.1 \mathrm{~mm} \times 70.1 \mathrm{~mm} \times 70.1 \mathrm{~mm})$. The curing time in this study was set as 7,28 , and 60 days. The detailed statistics of variables of CPB are given in Table 1. A total of 435 specimens were completed, and they were used for obtaining the

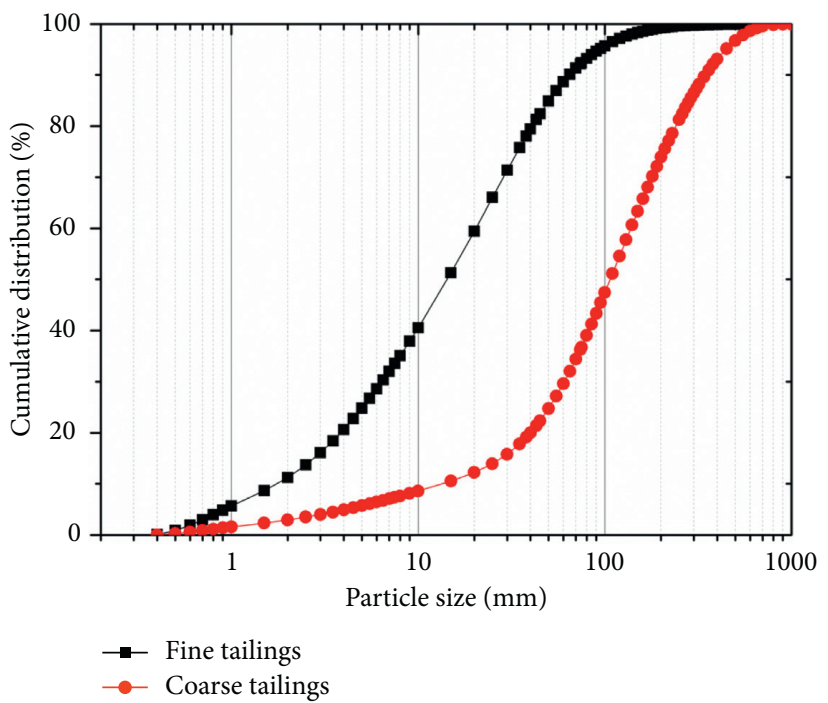

FIgURE 1: Grain size distribution of coarse and fine tailings.

UCS values by conducting unconfined compressive tests according to ASTM C 39.

\subsection{Model of Evolutionary Support Vector Machine (BSVM)}

2.2.1. Support Vector Machine (SVM). SVM is normally applied for classifying the samples by the hyperplanes [26]. When the hyperplane can make a large margin in two classes, the vectors corresponding to the hyperplanes are support vectors. The schematic diagram of the SVM is depicted in Figure 2.

Generally, the hyperplane equation is as follows:

$$
f(\mathbf{x})=\mathbf{w}^{T} g(\mathbf{x})+b,
$$

where $\mathbf{w}$ means an $\mathrm{m}$-dimensional vector; $b$ denotes the bias term; and when $\mathbf{w}$ and $b$ are obtained, the $x$ can be classified by the sign of $f(\mathrm{x})$.

For linear separable data, the following equation can be concluded as follows:

$$
y_{i}\left(\mathbf{w}^{T} g(\mathbf{x})+b\right)-1 \geq 0 .
$$

The support vectors are on the hyperplane:

$$
y_{i}\left(\mathbf{w}^{T} g(\mathbf{x})+b\right)=1 .
$$

To minimize the $\|w\|^{2}$, the hyperplane can be found $(\|w\|$ is the Euclidean norm of $\mathrm{w}$ ).

2.2.2. Beetle Antennae Search (BAS). BAS is a very famous metaheuristic algorithm, which is proposed recently [20]. It can be used for global optimization problems. Nowadays, BAS has been widely utilized in obtaining hyperparameters in machine learning algorithms [20,21]. In this algorithm, it simulated the beetles' behavior, and the objective of its antennae is to find the odor with high concentration. A typical flow chart of BAS is shown in Figure 3.

In this study, the hyperparameters of SVM (C, penalty coefficient and $\gamma$, kernel parameter) were tuned by BAS instead of trial-and-error methods. 
TABLE 1: Statistics of influencing variables.

\begin{tabular}{lcccc}
\hline Variable & Min & Max & Mean & Standard deviation \\
\hline Coarse tailings-cement ratio (T/C) & 4 & 10 & 7 & 2.2 \\
Solids-water ratio (S/W) & 0.68 & 0.72 & 0.7 & 0.02 \\
Fine tailings percentage (FTP) & 0 & 0.2 & 0.11 & 0.07 \\
Curing time & 7 & 60 & 31.6 & 21.8 \\
\hline
\end{tabular}

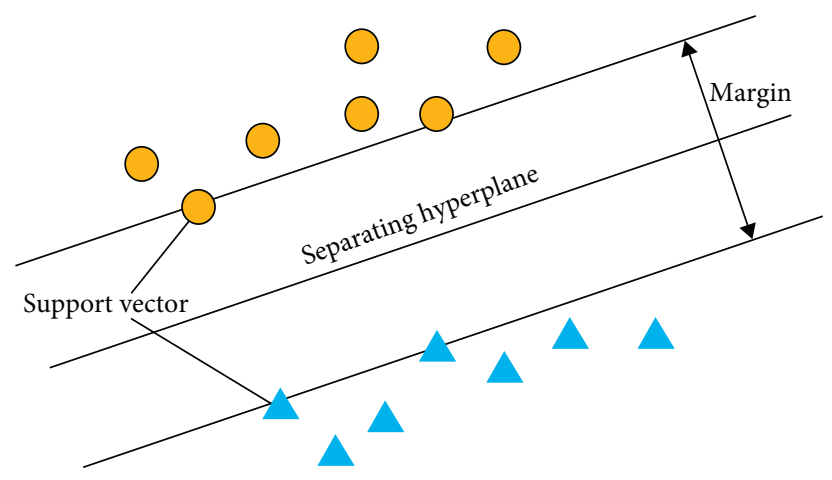

Figure 2: Diagram of SVM.

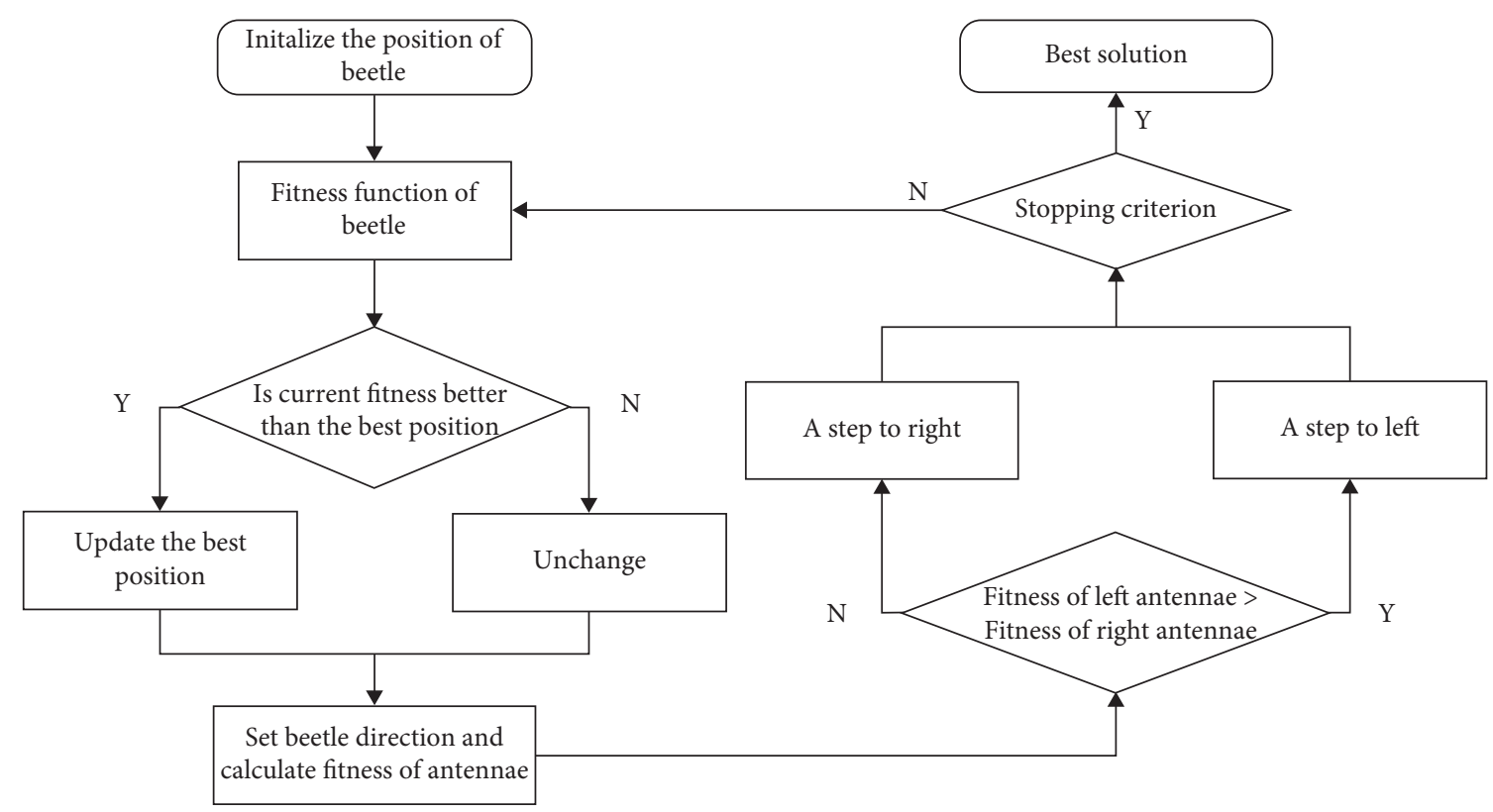

Figure 3: Flow chart of BAS.

2.3. Performance Evaluation. According to the suggestion in previous studies, the training dataset and testing dataset are split into $70 \%$ dataset and $30 \%$ dataset, respectively. A 10-fold cross- validation method was applied. The correlation coefficient $(R)$ and root-mean-square error (RMSE) for evaluating the performance of the established model are defined as follows:

$$
\begin{aligned}
R & =\frac{\sum_{i=1}^{N}\left(y_{i}^{*}-\overline{y^{*}}\right)\left(y_{i}-\bar{y}\right)}{\sqrt{\sum_{i=1}^{N}\left(y_{i}^{*}-\overline{y^{*}}\right)} \sqrt{\sum_{i=1}^{N}\left(y^{i}-\bar{y}^{2}\right)}}, \\
\text { RMSE } & =\sqrt{\frac{1}{N} \sum_{i=1}^{N}\left(y_{i}^{*}-y_{i}\right)^{2}},
\end{aligned}
$$




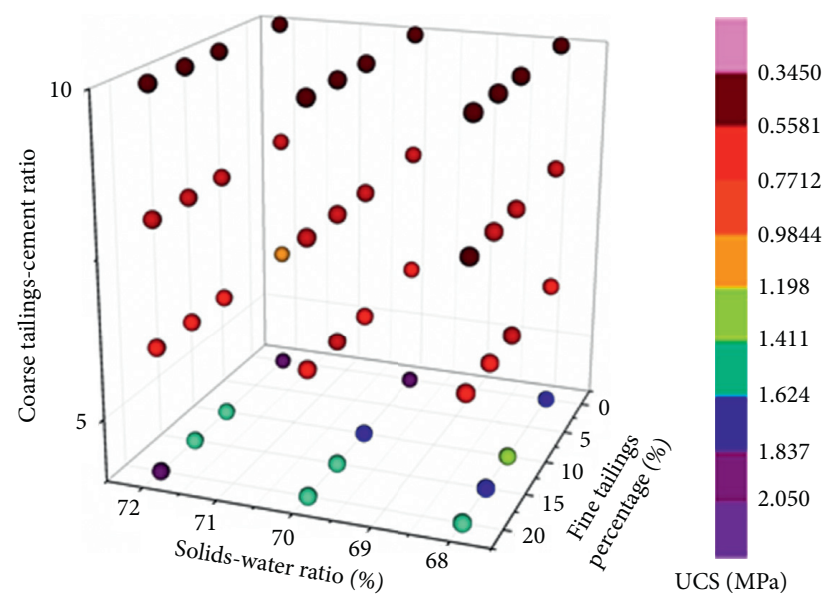

(a)

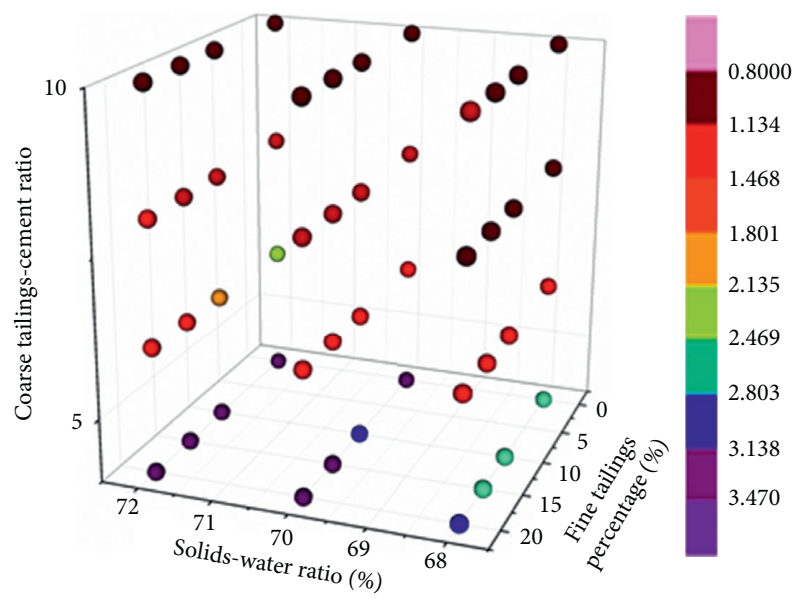

(b)

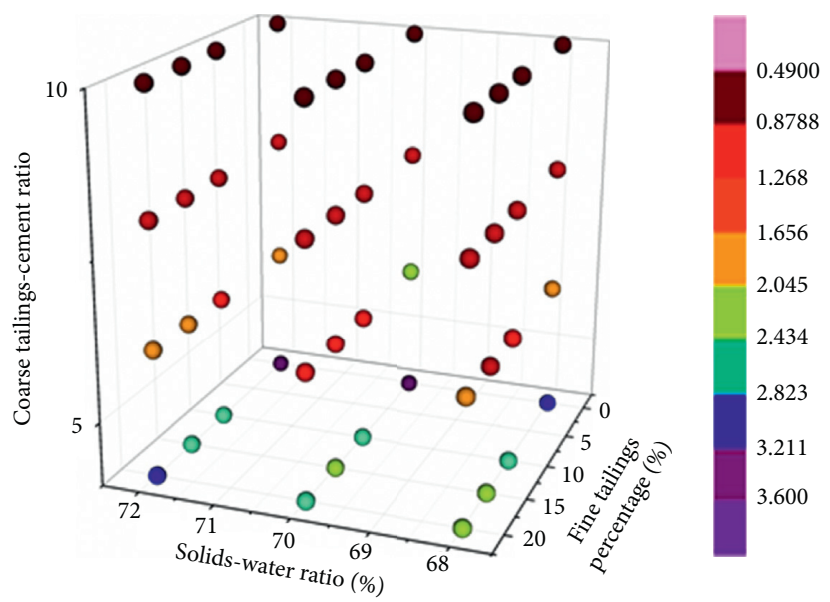

(c)

Figure 4: UCS test results of CPB in 4D: (a) 7 days; (b) 28 days; (c) 60 days.

where $N$ means the numbers in the dataset; $y_{i}^{*}$ and $y_{i}$ are the expected values and real values, respectively; and $\bar{y}$ and $\overline{y^{*}}$ indicate the mean predicted values and mean actual values, respectively.

\section{Results and Discussion}

3.1. Results of UCS of CPB. Figure 4 shows the UCS of CPB combined with different variables under different curing times. It can be seen that the coarse tailings-cement ratio is the main index for determining the strength of CPB. With the increase of coarse tailings-cement ratio, the UCS of CPB increased obviously. Similarly, the UCS of CPB improved with the increase of the solids-water ratio. However, in terms of the effect of fine tailing percentage on CPB strength, it depends on the solids-water ratio. Specifically, when the solids-water ratio is between $68 \%$ and $70 \%$, with the increase of the fine tailing percentage, the UCS of CPB increased to the peak values and then declined. When the solids-water ratio is $72 \%$, the UCS of CPB decreased slightly with the increase of fine tailing percentage. The curing time played a positive effect on the increase in the strength of $\mathrm{CPB}$, which is consistent with the previous studies.
3.2. Results of Hyperparameter Tuning. In this study, BAS is applied to tune hyperparameters of SVM on the training set. RMSE is selected as the objective function. Figure 5 shows the RMSE versus iteration curve. It can be seen that RMSE decreases significantly and is stable after 15 iterations, indicating that the BAS is efficient in tuning hyperparameters. The final hyperparameters of SVM are tabulated in Table 2.

3.3. Assessment of the Established Model. Figure 6 shows the correlation between predicted UCS values and actual UCS values on the training and test sets. A nearly linear relationship is observed with $R$ values of 0.9701 and 0.973 on the training and test sets, respectively, indicating that the proposed SVM model can establish the relationship between the UCS of CPB and its influencing variables successfully. Besides, the low and similar RMSE values on the training $(0.1798)$ and test $(0.2674)$ sets suggest that no underfitting or overfitting phenomena are produced.

3.4. Analysis of the Variable Importance. The relative importance of the input variables is calculated using global sensitivity study, as shown in Figure 7. It can be observed 


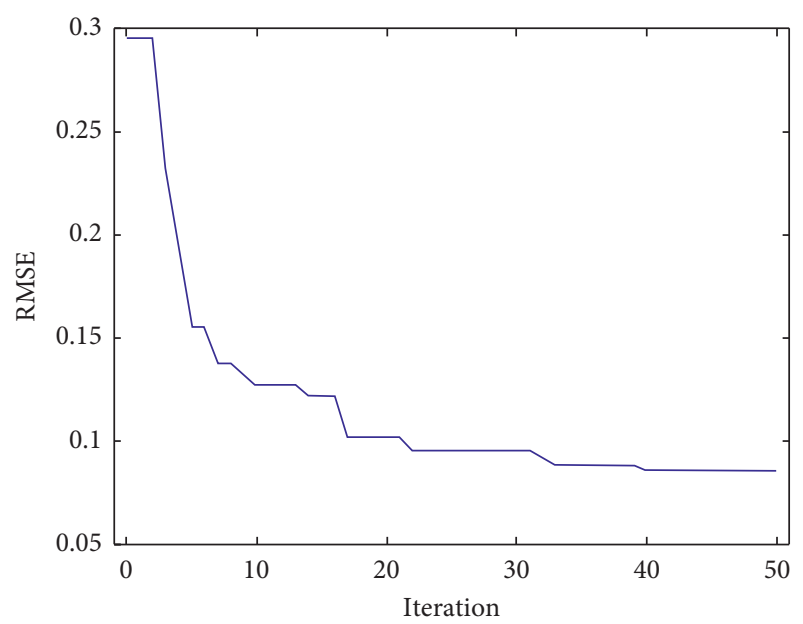

FIgURE 5: RMSE versus iterations.

TABLE 2: The obtained hyperparameters of RF.

\begin{tabular}{lccc}
\hline Parameters & Empirical scope & Initial & Results \\
\hline C & {$[0.1,1000]$} & 14 & 65 \\
$\Gamma$ & {$[0.001,100]$} & 14 & 1.01 \\
\hline
\end{tabular}

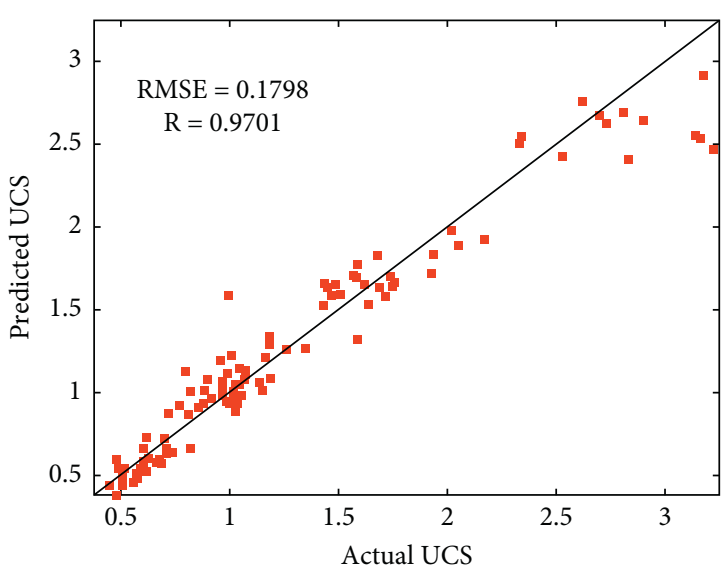

(a)

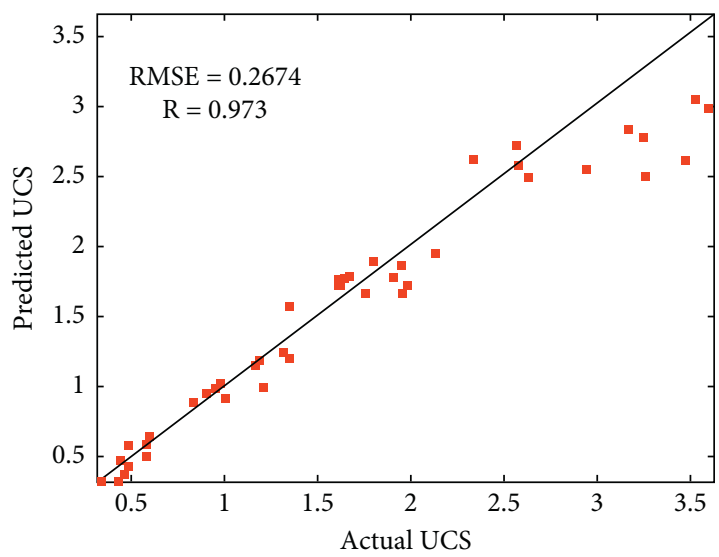

(b)

FIgURE 6: Comparison of UCS values. (a) Training dataset. (b) Testing dataset.

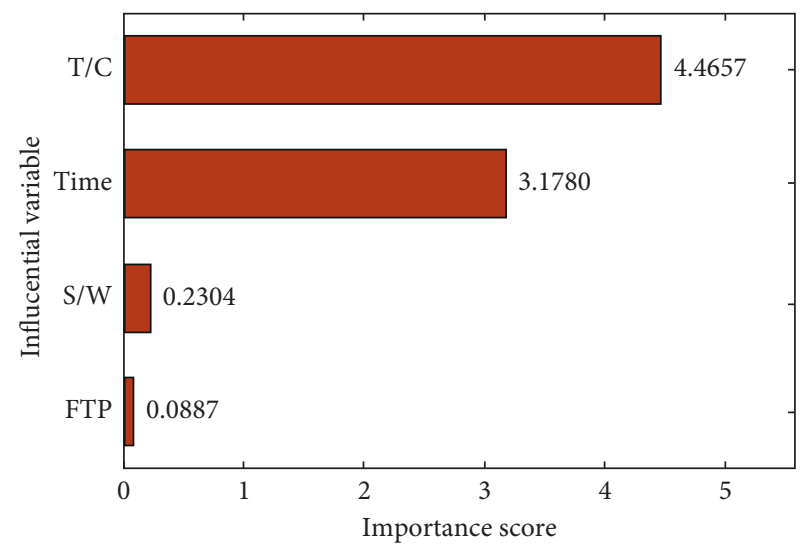

FIgURE 7: Variable importance of CPB based on the BSVM.

that the coarse tailings-cement ratio has the most significant influence on the UCS of CPB with an influencing score of 4.46 , followed by curing time (3.178) and solids-water ratio (0.23), while fine tailings percentage is the least sensitive variable with an influence score of 0.088 . This result agrees well with the previous study. It should be noted that the importance score is obtained by the dataset used in this paper. More accurate results can be obtained if more data samples are included in the dataset in the future.

\section{Conclusions}

This study uses the BSVM for predicting the UCS of CPB. The hyperparameters of SVM are tuned by BAS. The BSVM can establish the relationship between the UCS of CPB and its influencing variables successfully, indicated by high correlation coefficients on the training (0.97) and test (0.973) sets. Also, the calculated variable importance by sensitivity analysis shows the coarse tailings-cement ratio is the most important variable to UCS.

In future work, the dataset will be enlarged by including more influencing variables and samples to improve the generalizability of the proposed model. Also, a graphical user interface will be implemented to facilitate the use of the model in designing CPB mixtures.

\section{Data Availability}

The Microsoft Excel Worksheet data used to support the findings of this study are available from the corresponding author (liguichen@cumt.edu.cn) upon request.

\section{Conflicts of Interest}

The authors declare that there are no conflicts of interest.

\section{Acknowledgments}

This study was financially supported by the "National Key Research and Development Program (grant number: 2016YFC0600901)" and "National Natural Science Foundation of China (grant numbers: 51574224 and 51704277)". The authors are grateful to Huaibei Mining (Group) Co. Ltd. 
The author thanks Dr. Zuqi Wang for her encouragement and help.

\section{References}

[1] C. Qi and A. Fourie, "Cemented paste backfill for mineral tailings management: review and future perspectives," Minerals Engineering, vol. 144, p. 106025, 2019.

[2] Y. Sun, G. Li, and J. Zhang, "Investigation on jet grouting support strategy for controlling time-dependent deformation in the roadway," Energy Science \& Engineering, vol. 8, no. 4, pp. 1-8, 2020.

[3] Y. Sun, G. Li, J. Zhang, and D. Qian, "Experimental and numerical investigation on a novel support system for controlling roadway deformation in underground coal mines," Energy Science \& Engineering, vol. 8, no. 2, pp. 490-500, 2020.

[4] Y. Sun, G. Li, J. Zhang, and D. Qian, "Stability control for the rheological roadway by a novel high-efficiency jet grouting technique in deep underground coal mines," Sustainability, vol. 11, no. 22, p. 6494, 2019.

[5] H. Basarir, Y. Sun, and G. Li, "Gateway stability analysis by global-local modeling approach," International Journal of Rock Mechanics and Mining Sciences, vol. 113, pp. 31-40, 2019.

[6] Y. Sun, G. Li, and J. Zhang, "Developing hybrid machine learning models for estimating the unconfined compressive strength of jet grouting composite: a comparative study," Applied Sciences, vol. 10, no. 5, p. 1612, 2020.

[7] Q. Chen, Q. Zhang, C. Qi, A. Fourie, and C. Xiao, "Recycling phosphogypsum and construction demolition waste for cemented paste backfill and its environmental impact," Journal of Cleaner Production, vol. 186, pp. 418-429, 2018.

[8] Q. Chen, Q. Zhang, A. Fourie, and C. Xin, "Utilization of phosphogypsum and phosphate tailings for cemented paste backfill," Journal of Environmental Management, vol. 201, pp. 19-27, 2017.

[9] Q.-S. Chen, Q.-L. Zhang, A. Fourie, X. Chen, and C.-C. Qi, "Experimental investigation on the strength characteristics of cement paste backfill in a similar stope model and its mechanism," Construction and Building Materials, vol. 154, pp. 34-43, 2017.

[10] C.-c. Qi, "Big data management in the mining industry," International Journal of Minerals, Metallurgy and Materials, vol. 27, no. 2, pp. 131-139, 2020.

[11] D. Wang, Q. Zhang, Q. Chen, C. Qi, Y. Feng, and C. Xiao, "Temperature variation characteristics in flocculation settlement of tailings and its mechanism," International Journal of Minerals, Metallurgy and Materials, 2020.

[12] C. Qi, A. Fourie, Q. Chen, and Q. Zhang, “A strength prediction model using artificial intelligence for recycling waste tailings as cemented paste backfill," Journal of Cleaner Production, vol. 183, pp. 566-578, 2018.

[13] Z. Zhang, E. Wang, and N. Li, "Fractal characteristics of acoustic emission events based on single-link cluster method during uniaxial loading of rock," Chaos, Solitons \& Fractals, vol. 104, pp. 298-306, 2017.

[14] Z. Zhang, E. Wang, Y. Zhang et al., "Analysis on the timefrequency characteristics of ultrasonic waveform of coal under uniaxial loading," Fractals, vol. 27, no. 06, pp. 19501001950123, 2019.

[15] S. Xu, F. T. Suorineni, K. Li, and Y. Li, "Evaluation of the strength and ultrasonic properties of foam-cemented paste backfill," International Journal of Mining, Reclamation and Environment, vol. 31, no. 8, pp. 544-557, 2017.
[16] W. Xu, X. Tian, and P. Cao, "Assessment of hydration process and mechanical properties of cemented paste backfill by electrical resistivity measurement," Nondestructive Testing and Evaluation, vol. 33, no. 2, pp. 198-212, 2018.

[17] J. Zhang, G. Ma, Y. Huang, J. sun, F. Aslani, and B. Nener, "Modelling uniaxial compressive strength of lightweight selfcompacting concrete using random forest regression," Construction and Building Materials, vol. 210, pp. 713-719, 2019.

[18] C. Qi, A. Fourie, and Q. Chen, "Neural network and particle swarm optimization for predicting the unconfined compressive strength of cemented paste backfill," Construction and Building Materials, vol. 159, pp. 473-478, 2018.

[19] J. Sun, J. Zhang, Y. Gu, Y. Huang, Y. Sun, and G. Ma, "Prediction of permeability and unconfined compressive strength of pervious concrete using evolved support vector regression," Construction and Building Materials, vol. 207, pp. 440-449, 2019.

[20] Y. Sun, J. Zhang, G. Li, Y. Wang, J. Sun, and C. Jiang, "Optimized neural network using beetle antennae search for predicting the unconfined compressive strength of jet grouting coalcretes," International Journal for Numerical and Analytical Methods in Geomechanics, vol. 43, no. 4, pp. 801813, 2019.

[21] Y. Sun, J. Zhang, G. Li et al., "Determination of Young's modulus of jet grouted coalcretes using an intelligent model," Engineering Geology, vol. 252, pp. 43-53, 2019.

[22] J. Zhang, D. Li, and Y. Wang, "Toward intelligent construction: prediction of mechanical properties of manufactured-sand concrete using tree-based models," Journal of Cleaner Production, vol. 258, Article ID 120665, 2020.

[23] Y. Sun, G. Li, J. Zhang, and D. Qian, "Prediction of the strength of rubberized concrete by an evolved random forest model," Advances in Civil Engineering, vol. 2019, Article ID 5198583, 7 pages, 2019.

[24] L. Orejarena and M. Fall, "The use of artificial neural networks to predict the effect of sulphate attack on the strength of cemented paste backfill," Bulletin of Engineering Geology and the Environment, vol. 69, no. 4, pp. 659-670, 2010.

[25] L. Orejarena and M. Fall, "Artificial neural network based modeling of the coupled effect of sulphate and temperature on the strength of cemented paste backfill," Canadian Journal of Civil Engineering, vol. 38, no. 1, pp. 100-109, 2011.

[26] C. Qi, Q. Chen, A. Fourie, and Q. Zhang, "An intelligent modelling framework for mechanical properties of cemented paste backfill," Minerals Engineering, vol. 123, pp. 16-27, 2018. 